This item is the archived peer-reviewed author-version of:

\title{
Public sector antiretroviral treatment programme in South Africa : health care workers' attention to mental health problems
}

\section{Reference:}

Pappin Michele, Wouters Edwin, Booysen Frederik L.R., Lund Crick.- Public sector antiretroviral treatment programme in South Africa : health care workers' attention to mental health problems

AIDS care - ISSN 0954-0121 - 27:4(2015), p. 458-465

Full text (Publishers DOI): http://dx.doi.org/doi:10.1080/09540121.2014.969674

Handle/Permalink: http://hdl.handle.net/10067/1230370151162165141 


\title{
Public sector ART programme in South Africa: Health care workers' attention to mental health problems
}

Michele Pappin $^{\mathrm{a} *}$, Edwin Wouters ${ }^{\mathrm{b}}$, Frederik LR Booysen $^{\mathrm{c}}$, \& $_{\text {Crick Lund }}^{\mathrm{d}}$

${ }^{a}$ Centre for Development Support, University of the Free State, South Africa

${ }^{\mathrm{b}}$ Research Centre for Longitudinal and Life Course Studies (CELLO), University of Antwerp, Belgium and Centre for Health Systems Research, University of the Free State, South Africa

${ }^{c}$ Department of Economics, University of the Free State, South Africa

${ }^{\mathrm{d}}$ Alan J Flisher Centre for Public Mental Health, Department of Psychiatry and Mental Health, University of Cape Town, South Africa

\begin{abstract}
Although there is a high prevalence of anxiety and depression amongst people receiving ART, many patients are not screened, diagnosed or referred for mental health problems. This study aims to determine whether public sector health care workers in South Africa observe, screen, diagnose and refer ART patients that show symptoms of common mental disorders. It also aims to ascertain the extent of mental health training received by public sector health care workers working in ART. The study was cross-sectional in design. Self-administered questionnaires were completed by 40 nurses and structured interviews were conducted with 23 lay workers across the five districts in the Free State between July 2009 and October 2009. STATA version 12 was used to perform statistical data analysis. The health care workers reported observing a high frequency
\end{abstract}


of symptoms of common mental disorders among public sector ART patients. While $70 \%$ of nurses screened and diagnosed, only $40 \%$ of lay workers screened and diagnosed patients on ART for a mental disorder. Health care workers who had received training in mental health were more likely to screen or diagnose a mental disorder, but only $14 \%$ of the workers had received such training. We recommend that health care workers should receive task specific training to screen and/or diagnose patients on ART for common mental disorders using the guidelines of the South African HIV Clinicians Society. A positive diagnosis should be referred to a healthcare practitioner for appropriate evidence-based treatment in the form of medication or psychotherapy.

Keywords: health care workers; ART; mental health; screening; training; South Africa 


\section{Introduction}

Globally, anxiety and depression are highly prevalent among patients receiving antiretroviral treatment (ART) (Nogueira Campos, Crosland Guimarães, \& Remien, 2010; Horberg et al., 2008). The same is true in South Africa, where $30.6 \%$ and $25.4 \%$ of patients on ART have symptoms of anxiety and depression, respectively (Pappin, Wouters, \& Booysen, 2012). It has also been found that anxiety and depression are co-morbid with each other (Hasanah, Zaliha, \& Mahiran, 2011) and associated with non-adherence to ART (Horberg et al., 2008).

The introduction of ART has had a major impact on the illness course for people living with HIV, transforming their experience from living with a terminal illness to living with a chronic illness. ART however is a life-long commitment and requires adherence levels of $95 \%$ or more to prevent disease progression and premature death (Horberg et al., 2008; O'Cleirigh, Hart, \& James, 2008; Patterson et al., 2000). Treatment of a mental illness offers large benefits. Depressed patients receiving ART who are prescribed with and remain adherent to selective serotonin re-uptake inhibitors (SSRI) display significantly higher levels of ART adherence and better ART outcomes (Horberg et al., 2008).

Despite the obvious benefits of diagnosing and treating mental disorders in patients receiving ART, anxiety and depression remain under-detected in patients attending public health care facilities around the world (Kagee, Tsai, Lund, \& Tomlinson, 2013). In South Africa, approximately one in six people experience anxiety and/or depression, and the prevalence of a mental disorder has not decreased with the introduction of ART (Jonsson et al., 2013). Of concern is that three-quarters of South Africans who live with a mental disorder do not receive adequate treatment (Lund et al., 2012; Williams et al., 2008). 
Health system and patient factors contribute to the low detection and treatment of mental disorders in South Africa. The health system has insufficient human resources to meet the populations mental health needs. An additional 361 psychiatrist, 135 nurses, and 2937 psychological care providers, which include psychologists and social workers, are needed to meet the mental health demands of South Africans (Day, Gray, \& Budgell, 2011). Patient factors associated with low detection and treatment of mental disorders are poor mental health literacy and stigma associated with mental disorders (Sorsdahl, Stein, \& Lund, 2012).

A Cochrane review of 38 studies from low and middle income countries reported that the use of non-specialised health workers (NSHWs) may increase the number of adults who recover from anxiety and depression (Van Ginneken et al., 2013). The review was unable to draw conclusions as to what type of NSHW, or what format or intensity of mental health training, and supervision were most effective in addressing common mental disorders (Van Ginneken et al., 2013). Current research recommends that mental health training format must be specific to the task to be carried out by the NSHW, and all training for mental health must be accompanied by effective ongoing supervision (Mendenhall et al., 2014; Kakuma et al., 2011).

Researchers have recommended task shifting as a means of increasing the number of patients screened for common mental disorders, especially at ART clinics hereby bridging the gap between under detection and treatment (Kagee et al., 2013). However, little is known about the capacity of current public sector health care workers to screen, diagnose and refer ART patients with common mental disorders, or the state of current practice.

Against this background the Social Action Theory (SAT) has been used as a framework for this research and the theoretical framework against which to understand task-shifting (Ewart, 1991). SAT is been advocated as the model to be used in guiding HIV behavioural research 
(Traube, Holloway, \& Smith, 2011). The model has three dimensions; public health (contextual dimension), task shifting (process dimension) and health outcome (action state dimension). The diagram below explains the adapted SAT model against the introduction discussed above (Figure 1).

[Insert Figure 1 here]

The aims of the paper are: (1) to determine the frequency with which public sector health care workers observe symptoms of anxiety, depression or other mental illnesses among ART patients, (2) to determine whether public sector health care workers screen, diagnose and refer ART patients suffering from anxiety, depression or other mental illnesses, and (3) to determine the extent of mental health training received by public sector ART health care workers. 


\section{Methods}

\section{Setting and participants}

This cross-sectional study was conducted between July 2009 and October 2009 as part of a larger panel study entitled Free State Effective AIDS Treatment and Support (FEATS). Health care workers in this study refer to nurses and lay workers. The ART manager working at a facility provided the fieldwork manager with a list of all nurses and lay health workers who provide services to patients receiving ART. Nurses were professionally trained and registered with the HPCSA. Nurses initiated ART and did routine follow-up appointments with patients. Lay workers performed HIV counselling and testing. All nurses and lay workers were purposively recruited into the study from 16 public sector antiretroviral sites across all five districts of the Free State Province. No record was kept of nurses and lay workers who did not consent to participate in the study, thus refusal rates of nurses and lay workers are not known.

\section{Procedures}

Nurses who gave written informed consent to participate in the study were requested by fieldwork managers to complete a six-page self-administered questionnaire. For those nurses who did not complete the questionnaire, fieldworkers completed the questionnaire by interviewing the nurse. Lay workers were interviewed in person by trained fieldworkers after providing written informed consent. The questionnaire for the nurses and structured interviews with the lay workers contained questions on the following topics: socio-demographic information (age, gender and education), health-related qualifications, current position held, formal training on mental health and short training courses on mental health. The following 
questions pertaining to the screening, diagnosis and referral of ART patients with symptoms of a mental disorder were also included: (1) do you observe signs and symptoms of anxiety, depression or other mental illness among ART clients? (2) do you screen or diagnose clients on ART for anxiety, depression or other mental illness? and (3) do you ever refer ART clients potentially suffering from anxiety, depression or other mental illness? The response options for question (1) were 'never', 'occasionally', 'frequently' and 'all the time', while the response options for questions (2) and (3) were 'yes' or 'no'.

\section{Data analysis}

STATA version 12 was used to perform statistical data analysis. First, descriptive characteristics of the health care workers were generated. Then bivariate analyses were conducted. Exact tests were used to assess the statistical significance of the differences in observing, screening, diagnosing and referring ART patients for symptoms of anxiety, depression or other mental illnesses across district and health care worker type. Subsequently, descriptive statistics of mental health training received by the health care workers were produced. Next, two sets of bivariate analyses using exact tests were run: (1) to assess whether there was a statistically significant difference in the observation, screening, diagnosis and referral of ART patients showing symptoms of anxiety, depression or other mental illnesses between health care workers who had received training in mental health and those who had not, (2) to assess there was a statistically significant difference in the observation, screening, diagnosis and referral of ART patients showing symptoms of anxiety, depression or other mental illnesses between type (nurse versus lay worker) of health care workers who had received training in mental health. Finally, multivariate analyses were run, however due to the small sample sizes the models were not 
significant and therefore not discussed in the result section. The data analysis includes all observations for which there was no missing data.

\section{Ethical considerations}

This study was approved by the Ethics Committee of the Faculty of Health Sciences at the University of the Free State [ETOVS 145/07 DOH-27-0907-2025]. Participation in the study was voluntary, and written informed consent was obtained from all participants. 


\section{Results}

\section{Health care worker characteristics}

Sixty three participants were recruited into the study, comprising 40 nurses and 23 lay workers. Ninety percent (56) of the study participants were female. The mean and median age was 41 years (Inter-Quartile Range (IQR): 33 - 46). Nine percent (6) of the respondents had some secondary education, $29 \%$ (18) held a matric certificate, $48 \%$ (30) a post-matric qualification, and 14\% (9) a bachelor's degree. Except for the lay workers and one nurse who was a part-time employee, all of the nurses were full-time employees. The mean number of years that the participants had been working at the specific health care facility was 5.3, and the median was 3.4 (IQR: $1.9-6.0)$. The mean number of years of experience that study participants had in the public health sector was 11.3 , and the median was 8.6 (IQR: $4.3-16.0$ ).

\section{Bivariate analysis}

The difference between the five districts in terms of the participants' observation, screening, diagnosis and referral of patients on ART for a mental health disorders was not statistically significant. The frequency with which the two types of participants (nurses and lay workers) observed signs and symptoms of anxiety, depression or other mental illnesses in ART patients did show a statistically significantly difference, but only a weak one (Fisher's exact $=5.7 ; \mathrm{p}=$ 0.08). Only one (2.6\%) nurse and one (4.4\%) lay worker reported never having observed signs or symptoms of anxiety, depression or other mental illnesses. A large percentage of nurses observed symptoms of anxiety and depression occasionally (61.5\%), while lay workers were more likely to observe symptoms frequently $(39.1 \%)$ or all of the time $(26.1 \%)$ (Table 1$)$. 
[Insert Table 1 here]

Fifty eight percent (35) of the participants reported having screened or diagnosed patients on ART for anxiety, depression or other mental illnesses. A higher percentage of nursing staff $(70.3 \%)$ than lay workers $(39.1 \%)$ had screened or diagnosed patients for anxiety, depression or other mental illnesses (Fisher's exact $=5.7, \mathrm{p}=0.03)($ Table 2$)$.

[Insert Table 2 here]

Eighty eight percent (51) of the participants reported having referred ART patients who were potentially suffering from anxiety, depression or other mental illnesses for treatment. All lay workers reported having referred patients, compared with $80.6 \%$ of the nursing staff (Fisher's exact $=4.9 ; \mathrm{p}=0.04)$

[Insert Table 3 here]

Only $6(10.0 \%)$ participants (four nurses and two lay workers) had received training in mental health as part of their formal ART education. For three additional participants (4.7\%) (one nurse and two lay workers), the last training they had attended in the preceding six months had been on mental health and had been presented by either a non-governmental organisation or the Department of Health. 
There was a statistically significant difference between participants who had received training in mental health and those who had not in the frequency of observing signs and symptoms of anxiety, depression or other mental illnesses in ART patients (Fisher's exact $=$ 10.6; $\mathrm{p}=0.01)$. Health care workers who had not received training in mental health were more likely to observe signs and symptoms of anxiety, depression or other mental illness occasionally (54.7\%) and all of the time (20.8\%), while nursing staff who had received training were more likely to observe symptoms frequently $(66.7 \%)$ (Table 4).

[Insert Table 4 here]

The relationship between having received training in mental health and the screening or diagnosis of patients receiving ART potentially suffering from anxiety, depression or other mental illnesses was weakly statistically significant (Fisher's exact $=4.1 ; \mathrm{p}=0.07)$. Health care workers who had received training in mental health screened or diagnosed a larger percentage of patients potentially suffering from anxiety, depression or other mental illness compared to health care workers who had not received training in mental health (88.9\% versus 53.0\%) (Table 5).

[Insert Table 5 here]

The relationship between having received training in mental health and referral of ART patients showing symptoms of anxiety, depression or other mental illnesses was not statistically significant (Fisher's exact $=0.009 ; \mathrm{p}=1.0$ ). The percentage of health care workers with and 
without training who referred patients potentially suffering with anxiety, depression or other mental illness was similar (88.9\% versus $87.7 \%)$ (Table 6).

[Insert Table 6 here]

The relationship between the type of health care worker who received training in mental health and the frequency of observing signs and symptoms of anxiety, depression or other mental illnesses was not statistically significant (Fisher's exact $=0.44 ; \mathrm{p}=0.23$ ). The nurses who received training in mental health observed signs and symptoms occasionally (40\%) and frequently $(60 \%)$, while $25 \%$ of lay workers with training never observed signs and symptoms of anxiety, depression or other mental illnesses, and $75 \%$ of lay workers frequently observed patients with anxiety, depression or other mental illness (Table 7).

[Insert Table 7 here]

The relationship between the type of health care worker who received training in mental health and screening or diagnosis of anxiety, depression or other mental illnesses was not statistically significant (Fisher's exact $=0.44 ; p=0.24$ ). However, all nurses who had received training in mental health screened or diagnosed patients with anxiety, depression or other mental illnesses compared to seventy five percent of the lay workers (Table 8).

[Insert Table 8 here] 
The relationship between the type of health care worker who received training in mental and the referral of ART patients showing symptoms of anxiety, depression or other mental illnesses was not statistically significant (Fisher's exact $=0.56 ; \mathrm{p}=0.34$ ). Eighty percent of nurses and all lay workers referred patients showing symptoms of anxiety, depression or other mental illness (Table 9).

[Insert Table 9 here] 


\section{Discussion}

Almost all of the health care workers (nursing staff and lay workers) working in public sector ART programmes in the Free State had observed signs or symptoms of anxiety, depression, stress or other mental illnesses in ART patients. More than half of the health care workers had screened or diagnosed patients potentially suffering from anxiety, depression or other mental illnesses, while almost ninety percent had referred patients for mental health problems.

Similarly, ARV nurses and counsellors who provided services to people living with HIV/AIDS in the Western Cape, South Africa, reported referring patients with a possible mental disorder to a colleague or mental health organisation outside the clinic rather than screening the patient themselves. The health care workers lack of self-confidence to screen and lack of training to screen for mental disorder were the reasons for their preference to refer patients (Mall, Sorsdahl, Swartz \& Joska, 2012). Thus, further research is needed to determine why almost all of the health care workers in the Free State had observed signs and symptoms of anxiety, depression or other mental illnesses among ART patients, but only half of them screened and diagnosed. Possible explanations might be that the ART patients' mental health symptoms were sub-clinical, or the health care workers did not have sufficient training to correctly diagnose and therefore refer ART patients showing signs or symptoms of mental illness (Lund et al. 2012; Mall, Sorsdahl, Swartz \& Joska, 2012).

Both nurses and lay workers reported observing a high frequency of signs and symptoms of anxiety, depression or other mental illnesses in ART patients. As expected given their higher level of training, a significantly higher percentage of nurses than lay workers had screened or 
diagnosed anxiety, depression or other mental illnesses. While the percentage of lay workers and nurses had referred ART patients for a potential mental disorder was similar.

A major concern raised by this study is that relatively few of the health care workers had received official training in mental health, despite the fact that health care staff are screening and diagnosing potential mental disorders in ART patients. Importantly, the few health care workers who had received training in mental health were more likely to screen and diagnose ART patients with mental health problems.

Of the few nurses and lay workers who had received training, all nurses had screened and diagnosed ART patients with anxiety, depression or other mental illness, compared to the three quarters of lay workers who screened and diagnosed. This finding must be treated with caution due to the small number of health care workers who received training in mental health. NSHWs in the Western Cape working with HIV/AIDS supported the proposal that screening of mental disorders be the function of lay workers/counsellors provided the lay workers/counsellors receive training which is task specific, a standard screening tool for screening mental disorders is used, and the NSHWs receive ongoing supervision (Mall et al., 2012). This finding shows promise for the task shifting agenda of the new national Mental Health Policy and Strategic Plan (Department of Health, 2013), which advocates for the use of nurses and lay health workers to detect, manage and appropriately refer people with mental health problems.

In the case of ART patients this is particularly pertinent, as mental health intervention have been shown to improved ART adherence and viral load in cases of depression (Horberg et al., 2008). Thus if this intervention can be scaled up, it has a large potential public health benefit in improving ART adherence and HIV outcomes in a country which carries a major HIV related burden of disease. 
Task shifting is being advocated because of the scarce human and financial resources for mental health in South Africa (Sorsdahl et al., 2012). The use of lay workers in treating common mental disorders shows promise in low- and middle- income countries through psychotherapy (Van Ginneken et al., 2013; Sorsdahl et al., 2012). But before a patient can be treated for a mental disorder there needs to be a diagnosis.

Mental disorders are often under-diagnosed (Johnson et al., 2013; Sorsdahl, Stein \& Lund, 2012). Since patients are unlikely to disclose their mental state, Johnson and colleagues recommend that patients on ART be screened using the three questions in the recently published clinical guideline 'Management of mental health disorders in HIV-positive patients receiving ART' (Johnson et al., 2013). We recommend that patients need to be screened by a lay worker for mental disorders prior to ART initiation, routinely over ART career, and when a patient has a detectable viral load, as a detectable viral load may be an indication of non-adherence to ART because of a mental disorder (Jonsson et al., 2013).

According to the guideline, if a patient responds to any of the three screening questions, the Patient Health Questionnaire (PHQ-9) should be administered to the patient by the lay worker. The PHQ-9 is used both to diagnose and to assess outcomes for the treatment of mental disorders (Jonsson et al. 2013). If a patient is diagnosed with a common mental disorder the patient is to be referred to an ART nurse for appropriate evidence-based treatment in the form of medication or psychotherapy. Nurses are not licenced to prescribe schedule 5 psychotropic medications in South Africa. A patient with a common mental disorder is to be reassessed by a lay worker every two weeks for the first six weeks diagnosis of the disorder.

\section{Limitations of the study}


This study had the following limitations. Firstly, health care workers were purposely sampled and not recruited into the study by means of open enrolment. Secondly, health care workers were only recruited from phase one health care facilities at which ART was rolled out in 2004 and 2005. Thus, the findings cannot be generalised to the entire Free State Province or beyond. Thirdly, no record was kept of participants who refused to participate in the study. Hence selection bias may have occurred. Fourthly, the study was based on self-reported information, so the findings may not be completely accurate. Fifthly, the amount of training in mental health may be underestimated, as the survey/interview questions only asked about formal training in mental health pertaining to ART and training received at the last course attended. Finally, meaningful statistical analysis was constrained due to the small sample sizes and the resultant lack of statistical power.

\section{Conclusion}

This study highlights the importance of training nurses and lay workers working with ART patients in mental health. Almost all nurses and lay workers working with patients receiving ART had observed signs and symptoms of anxiety and depression. However, while $70 \%$ of nurses screened and diagnosed, only $40 \%$ of lay workers screened and diagnosed patients on ART for a mental disorder. Only $14 \%$ of the nurses and lay workers had received training in mental health, but those who had received training were more likely to screen or diagnose ART patients with a possible mental illness. We recommend the implementation of the task-shifting model which advocates for the task specific training and use of nurses and lay health workers to 
screen, diagnose and appropriately refer patients with mental health problems. A positive diagnosis should be referred to a healthcare practitioner for appropriate evidence-based treatment in the form of medication or psychological therapy. Research which investigates the effectiveness of the task-shifting model and the appropriate type of mental health training required for lay workers as opposed to ARV nurses in public sector ART programmes is needed.

\section{Acknowledgements}

The study was funded by the Research Committee of the World Bank, The Bank Netherlands Program Partnership, the WB-DfiD 'Evaluation of the Community Response to HIV and AIDS', the Health Economics and AIDS Research Division (HEARD) at the University of KwazuluNatal, the University of the Free State and the National Research Foundation (NRF) of South Africa. CL is supported by the PRogramme for Improving Mental health carE (PRIME), funded by the Department for International Development (DFID), UK. The findings, interpretations and conclusions in this article are those of the authors and do not reflect the views of the funding agencies.

\section{Competing interest}

The authors declare that they have no financial or personal relationships which may have inappropriately influenced them while writing this article. 


\section{References}

Day, C., Gray, A. \& Budgell, E. (2011). Health related indicators. In Paradath, A. \& English, R. (Eds). South African health review (pp 119-248). Durban, South Africa: Health Systems Trust.

Department of Health. (2013). National Mental Health Policy Framework and Strategic Plan 2013-2020. Pretoria: Department of Health.

Ewart, C.K. (1991). Social action theory for Public Health Psychology. American Psychologist, 46(9), 931-946.

Hasanah, C.I., Zaliha, A.R., \& Mahiran, M. (2011). Factors influencing the quality of life in patients with HIV in Malaysia. Quality of Life Research, 20, 91-100. doi: 10.1007/s11136010-9729-y

Horberg, M.A., Silverberg, M.J., Hurley, L.B., Towner, W.J., Klein, D.B., Bersoff-Matcha, S., ... Kovach, D.A. (2008). Effects of depression and selective serotonin reuptake inhibitor use on adherence to highly active antiretroviral therapy and on clinical outcomes in HIVinfected patients. Journal of Acquired Immune Deficiency Syndrome, 47(3), 384-390.

Jonsson, G., Davies, N., Freeman, C., Joska, J., Pahad, S., Thom, R., Thompson, K., Woollet, N., Furin, J. \& Meintjies, G. (2013). Management of mental health disorders in HIV-positive patients. South African Journal of HIV medicine, 14(4),155-165. doi:10.7196/SAJHIVMED.995

Kagee, A., Tsai, A.C., Lund, C. \& Tomlinson, M. (2013). Screening for common mental disorders in low resource settings: reason for caution and way forward. International Health, 5, 11-14. doi:10.1093/inthealth/ihs004 
Kakuma, R., Minas, H., van Ginneken, N., Dal Poz, M.R., Desiraju, K., Morris, J.E., Saxena, S. \& Scheffler, R.M. (2011). Human resources for mental health care: current situation and strategies for action. Lancet, 378(5), 1654-1663. Doi:10.1016/S0140-6736(11)61093-3

Lund, C., Petersen, I., Kleintjes S., \& Bhana, A. (2012). Mental health services in South Africa: taking stock. African Journal of Psychiatry, 15(6), 402-405.

doi.org/10.4314/ajpsy.v15i6.48

Mall, S., Sorsdahl, K., Swartz, L. \& Joska, J. (2012). “I understand just a little...” Perspectives of HIV/AIDS service providers in South Africa of providing mental health care for people living with HIV/AIDS. AIDS Care, 24(3), 319-323.

doi.org/10.1080/09540121.2011.608790

Mendenhall, E., De Silva, M.J., Hanlon, C., Petersen, I., Shidhaye, R., Jordans, M.,...Lund, C. (2014). Acceptability and feasibility of using non-specialist health workers to deliver mental health care: Stakeholders perception from the PRIME district sites in Ethiopia, India, Nepal, South Africa, and Uganda. Social Science \& Medicine, 118. doi.org/10.1016/j.socscimed. 2014.07.057.

Mojtabai, R., Olfson, M., Sampson, N.A., Jin, R., Druss, B., Wang, P.S., Wells, K.B., Pincus, H.A. \& Kessler, R.C. (2011). Barriers to mental health treatment: results from the National Comorbidity Survey Replication. Psychological Medicine, 41, 1751-1761. doi:10.1017/S0033291710002291

Nogueira Campos, L., Crosland Guimarães, M.D., \& Remien, R.H. (2010). Anxiety and depression symptoms as risk factors for non-adherence to antiretroviral therapy in Brazil. AIDS and Behaviour, 14, 289 - 299. doi: 10.1007/s10461-008-9435-8 
O'Cleirigh, C., Hart, T.A. \& James, C.A. (2008). HIV and anxiety. In M.J. Zvolensky \& J.A. Smits (Eds), Anxiety in health behaviors and physical illness (pp 317-340). New York, NY: Springer Science \& Business Media.

Pappin, M., Wouters, E. \& Booysen, F. (2012). Anxiety and depression amongst patients enrolled in a public sector antiretroviral treatment programme in South Africa: cross sectional study. BMC Public Health, 12, 244. doi:10.1186/1471-2458-12-244

Paterson, D.L., Swindells, S., Mohr, J., Brester, M., Vergis, E.N., Squler, C., Wagener, M.M. \& Singh, N. (2000). Adherence to Proteas Inhibitor Therapy and Outcomes in Patients with HIV infection. Annals of Internal Medicine,133, 21-30.

Sorsdahl, K., Stein, D.J. \& Lund, C. (2012). Mental health services in South Africa: Scaling up and future directions. African Journal of Psychiatry, 15, 168-171. doi.org/10.4314/ajpsy.v15i3.21

Traube, D.E., Holloway, I.W., \& Smith, L. (2011). Theory development for HIV behavioural health: empirical validation of behaviour health models specific to HIV risk. AIDS Care, 23(6), 663-670. doi: 10.1080/095402121.2010.532532.

Van Ginneken, N., Tharyan, P., Lewin, S., Rao, G.N., Meera, S.M., Pain, J., Chandrashekar, S. \& Patel, V. (2013). Non-specialist health care worker interventions for care of a mental, neurological, and substance-abuse disorders in low- and middle-income countries (Review). Cochrane Database of Systematic Reviews, 11. doi:10.1002/14651858.CD009149.pub2.

Williams, D. R., Herman, A., Stein, D. J., Heeringa, S. G., Jackson, P. B., Moomal, H., \& Kessler, R. C. (2008). Twelve-month mental disorders in South Africa: Prevalence, service 
use and demographic correlates in the population-based South African Stress and Health Study. Psychological Medicine, 38, 211-220. doi:10.1017/S0033291707001420 